\title{
Research on Internet Model and Distributed Routing Algorithm
}

\author{
Feng Jie \\ Dalian Naval Academy, Dalian, China \\ Fdf8821@163.com
}

\author{
Liu Feng, Su Qi \\ Dalian Naval Academy, Dalian, China \\ liufeng@163.com
}

\begin{abstract}
The Internet model is built up and a distributed routing Algorithm is designed, whose time complexity is analyzed. The static network model (SNM) and dynamic network model (DNM) are established and path DB and its replace rules are put forward. The improved distributed routing Algorithm is designed and its time complexity is also analyzed.
\end{abstract}

Keywords-Internet Model; Distributed routing Algorithm; Time complexity; path DB; Replace rules

\section{INTRODUCTION}

Centralized routing algorithm is hard to apply to the present Internet. Because a billion computers are being connected to the network and the number of nodes is increasing at exponent level [1, 2]. Reference [3,4] proposed QoS routing optimum policy based on Partheno-GA with Binary Coding of N-dimension matrix and simplified genetic operation, which solved convergence early problem and deleted cycle routing. It is reported that the method can be applied to any complex network topology. The Internet Model is first built up and distributed routing Algorithm A is designed, The static network model (SNM) and dynamic network model (DNM) are then established, and path DB and its replace rules are put forward. The improved distributed routing Algorithm A+ is designed and its time complexity is finally also analyzed.

\section{INTERNET MODEL AND A Distributed ROUTING ALGORITHM}

When internet model is desiged a simple structure frame is applied, which uses a 2-dimension space grid and whose edge is suppoused to be directed. Therfor, it is composed of a grid point set GS of an exact square $n \times n$, $G S=\{(i, j): i \in\{1,2, \ldots, n\}, j \in\{1,2, \ldots, n\}\}$, and the distance of two nodes $(i, j)$ and $(k, l)$ is defined as $d((i, j),(k, l))=|k-i|+|l-j|$, whose number is steps of seperating digital grid. For a constant number $p>1$, node $u$ is connected with the other nodes which are called local contacts. For $q \geq 0$ and $r \geq 0$, a directed edge from node $u$ to other node $q$ is constructed by independent random test which is called a remote or long contact. The possibility proportional of a directed edge from $u$ to final node $v$ is $[d(u, v)]^{-r}$, which is called reverse $r-$ power distribution. when $p$ and $q$ are contants, Internet models of a series of parameters are obtained through adjusting power $r$ : when $r=0$, an independent remote contact can be obtained. When $\boldsymbol{r}$ increases, a remote contact of a node is become more and more converged near the grid. $\boldsymbol{r}$ is represented as a basic structure parameter, which has a general network meaning. Two-dimensional grid network with local contacts and long contacts is shown in fig.1.

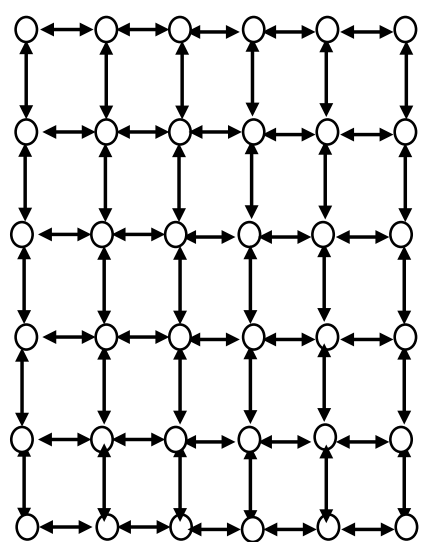

(a)

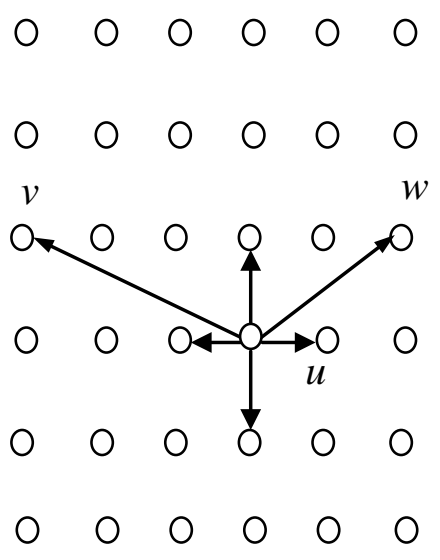

(b) (a) Two-dimensional grid network with $n=6, p=1$, and $q=0$.

(b) The contacts of a node $u$ with $p=1$ and $q=2 . v$ and $w$ are the two long-range contacts.

Fig. 1 Two-dimensional grid network with local contacts and long contacts

Starting from source node $s$ to target node $t$, a message $M$ is transfered through at least possible steps. A distributed routing Algorithm is researched which a message 
$M$ can be transported by order from a present node to its local or remote contact. Especielly, a node $u$ which holds a message $M$ can obtain the following knowleges through some steps:

(1) Local contacts of all nodes;

(2) The address of target node $t$ in the grid;

(3) All node address and remote contacts $V$ or $W$ which is connected with the message $M$.

If an intermediate node is selected from source node $S$ to target node $t$, the expectation time of a distributed routing Algorithm is $E(t)$, which is according to $[d(u, v)]^{-r}$ and the expectation steps of transfering message can be found by the algorithm.

A kind of a distributed routing Algorithm (A) is described as follows:

Input: Source node $s$, target node $t$, and node number array $B(i, j)$;

Output: Path p from source node $S$ to target node $t$.

Step1: Initiation : $P=\{s\}, b=s$;

Step2: The next node $B(i, j)$ is randomly selected which is locally connected with $b$ and it is at most near to the target node $t, b=B(i, j)$, and $b$ and $P$ are not close cycle, $P=P \cup\{b\}$.

Step3: IF $\boldsymbol{b}=\boldsymbol{t}$ THEN GOTO Step4

ELSE GOTO Step2 ;

Step4: OUTPUT $(P)$;

Step5: END

It is key that limiting the algorithm only knows to use the local contact in the model. If one node knows all its local and remote contatcts, then the optimal path between two nodes can be found by broad first seach method. Therefore, the above knowleges (1) and (2) are used when the up bound is analyzed in the distributed routing algorithm A, but the above knowlege (3) is used when the low bound is analyzed in the distributed routing algorithm $\mathrm{A}$.

To understand the time complexity of the distributed routing algorithm A, some concepts and mathematical symbols are first introduced.

Definition 2.1 (Up bound) :

iff $\exists c$ and $\exists n_{0}, \quad \forall n \geq n_{0}, \quad$ satisfy $|f(n)| \leq c|g(n)|, \quad g(n)$ is called the up bound of $f(n)$, which is noted as $f(n)=O(g(n))$ 。

Definition 2.2 (Low bound): iff $\quad \exists c \quad$ and $\quad \exists n_{0}, \quad \forall n \geq n_{0} \quad, \quad$ satisfy $|f(n)| \geq c|g(n)|, \quad g(n)$ is called the low bound of $f(n)$, which is noted as $f(n)=\Omega(g(n))$.

Definition 2.3 (Up bound+ Low bound) :

iff $\exists c_{1}, \quad \exists c_{2}$ and $\exists n_{0}, \quad \forall n \geq n_{0}, \quad$ satisfy $c_{1}|g(n)| \leq|f(n)| \leq c_{2}|g(n)|, \quad g(n)$ is not only called the up bound of $f(n)$ but also the low bound of $f(n)$, which is noted as $f(n)=\theta(g(n))$.

The time complexity of the distributed routing algorithm A is presented as follows through three theorems, whose proofs refer to as [5,6].

Theorem 2.1 (Low bound) :

$\exists \mathrm{A}$ and $\exists \alpha_{0}$, it is dependent on $p$ and $q$ but independent of $n$, when $r=0$, the time complexity of any A is $E(t) \geq \alpha_{0} n^{2 / 3}$.

Theorem 2.2 (Up bound):

$\exists \mathrm{A}$ and $\exists \alpha_{2}$, when $r=2, p=q=1$, the time complexity of any A is $E(t) \leq \alpha_{2}\left(\log _{2} n\right)$.

Theorem 2.3 :

(a) supposed that $0 \leq r<2, \exists \alpha_{r}$, it is dependent on $p$ and $q$ but independent of $n$, the time complexity of any $\mathrm{A}$ is $E(t) \geq \alpha_{r} n^{(r-2) / 3}$;

(b) supposed that $r>2, \exists \alpha_{r}$, it is dependent on $p$ and $q$ but independent of $n$, the time complexity of any A is $E(t) \geq \alpha_{r} n^{(r-2) /(r-1)}$.

\section{INTERnet Routing MODEL AND PATH DB}

The static network model (SNM) and dynamic network model (DNM) are first established and path DB and its replace rules are put forward in this section.

\section{Definition 3.1}

The static network model (SNM) is called as once the topology of the network $G(V, E, Q)$ is determined, in which $V=\{1,2, \ldots, N\}$ is node set and $E \subseteq V \times V$ is a link set of the network; the QoS parameters of $E$ are determined, in which QoS parameters mainly include band breadth $b_{d}$, time delay $t_{d}$, time delay jitter $t_{j}$, package lost rate $p_{l}$, hop $h_{c}$ and path cost $p_{c}$ etc., which is represented as $Q=\left\{w_{i j} \mid(i, j) \in E, i, j=1,2, \ldots N\right\}$ 
$w_{i j} \in\left\{b_{d}, t_{d}, t_{j}, \quad p_{l}, h_{c}, p_{c}\right\}$. i.e., for every link $(i, j) \in E$, it is the weight of link $(i, j) \in E$. These QoS parameters are not changed according to time $\mathrm{T}$.

\section{Definition 3.2}

The dynamic network model (DNM) is called as once the topology of the network $G(V, E, Q)$ is determined, in which node $V$, link $E$ and its QoS parameters are changed according to time $\mathrm{T}$, in which QoS parameters mainly include band breadth $b_{d}$, time delay $t_{d}$, time delay jitter $t_{j}$, package lost rate $p_{l}$, hop $h_{c}$ and path cost $p_{c}$ etc.. Therefore the DNM is represented as $G(V(t), E(t), Q(t))$, in which $V(t)=\{1,2, \ldots, N\}$ is node set and $E(t) \subseteq V(t) \times V(t)$ is link set of the DNM, $Q(t)=\left\{w_{i j}(t) \mid(i, j) \in E, i, j=1,2, \ldots N\right\}$, $w_{i j}(t) \in\left\{b_{d}(t), t_{d}(t), t_{j}(t), p_{l}(t), h_{c}(t), p_{c}(t)\right\}$, i.E. for every link $(i, j) \in E(t)$, it is a function set in $t \in\left[t_{0}, t_{m}\right] \in R$, which is the weight of link $(i, j) \in E$.

\section{Definition 3.3}

The Internet model $G(V, E, Q)$ is defined as Big World Network (BWN).

\section{Definition 3.4}

For every user $i(=1,2, \ldots, M)$ in Internet, he or she only visits a part nodes or network stations of BWN $G(V, E, Q)$ and accessing probability is $p$, those nodes or stations are called Small World Network (SWN) of user $i$ and $G\left(V_{i}, E_{i}, Q_{i}, p\right)$ is noted。

\section{Definition 3.5}

The routing selection of $S \rightarrow t$ in Internet is a process of finding a local contact $u$ or remote contact $v$ of $S \rightarrow t$.

According to Small World (SW) theory [6] , $G\left(V_{i}, E_{i}, Q_{i}, p\right)$ has Cluster property, whose cluster coefficient is

$$
C_{i}=E_{i} /\left(\begin{array}{l}
d_{i} \\
2
\end{array}\right)
$$

In which $d_{i}$ is degree of node $i$,which is edge number connected with node $\boldsymbol{i}$. This represents that a user $\boldsymbol{i}$ visits relatively fixed nodes. Particularly, because the node number of every user $\boldsymbol{i}$ visiting is limited and relatively fixed, we can store the path $P=\{s \rightarrow i \rightarrow j \rightarrow \ldots \rightarrow t\}$ of first computed and it is used next time, which can save a lot of routing calculation time. This is a typical example of save time by space. Obviously, developing with the capacity of disk and MEM this method can be implemented. Because the capacity of disk and MEM is limited, the stored old paths must be replaced according to some rules. Three replace rules are put forward as follows.

(1) FIFO rule

This is called First In First Out (FIFO) rule, when the DB of stored path $P$ is full then the earliest stored path $P$ is replaced. The mechanism of implementation the rule is simple and the timestamp is added to every path record.

(2) LRA rule

This is called Least Recently Access (LRA) rule, when the DB of stored path $P$ is full then the least recently access path $P$ is replaced. The mechanism of implementation the rule is more complex because the history of every visited path must be recorded and updated.

(3) TCD rule

This is called Topology Change Delete (TCD) rule, when the topology of the network $G(V, E)$ changes, then the changed path $P$ is replaced. For example, when the node $i$ in path $P$ is fault, the path cannot be used again, therefore, it is needed to be deleted from the DB.

\section{IMPROVEd Distributed ROUTING AlgorithM A+}

With the help of above replace rules and path $\mathrm{DB}$, an improved distributed routing Algorithm $\mathrm{A}^{+}$is formally described as follows.

Input: Source node $S$, target node $t$, node number array $B(i, j)$, and Path DB;

Output: Path p from source node $S$ to target node $t$.

Step1: Initiation: $P=\{s\}, b=s$;

Step2: IF $t \in D B$ THEN $\{P=D B(s \rightarrow t)$; GOTO Step5\};

Step3: The next node $B(i, j)$ is randomly selected which is locally connected with $b$ and it is at most near to the target node $t, b=B(i, j)$, and $b$ and $P$ are not close cycle, $P=P \cup\{b\}$; Step4: IF $b=t$ THEN \{ IF $D B \neq F U L L$

$$
\begin{aligned}
& \text { THEN }\{D B=D B \cup P \text {; } \\
& \text { GOTO Step5; \} } \\
& \text { ELSE } \\
& \text { \{ } D B=D B-O L D(P) \text { ； } \\
& D B=D B \cup P \quad ; \quad \text { GOTO } \\
& \text { Step5; \} } \\
& \text { ELSE GOTO Step3; }
\end{aligned}
$$

Step5: OUTPUT ( $P$ ) ; 


\section{Step6: END}

Note that in the improved distributed routing Algorithm $\mathrm{A}^{+}$, if the target $t$ and path $P$ are included in the DB, the source node $S$ directly uses the path which there is a remote contact $u$ in SW model and the remote contact is directly used to reach target $t$. When there not is the existed path in DB the improved distributed routing Algorithm $\mathrm{A}^{+}$is applied to find a new path $P$. Added new path to DB, it is needed to judge if DB is full. If it is full any rule in above three replace rules is applied to replace old path, i.e. $D B=D B-O L D(P)$. The meaning of the other statement is the same with the distributed routing Algorithm A .

The time complexity of the improved distributed routing Algorithm $\mathrm{A}^{+}$is simply analyzed. In the most ideal situation, the path is included in DB, which is only a search process, therefore, The time complexity of the Step2 of $\mathrm{A}^{+}$ is $\Omega(m / 2)$, in which $m$ is the number of the path in DB. In worst situation, i.e. there is not the path $P$ in $\mathrm{DB}$, the up bound of The time complexity of $\mathrm{A}^{+}$is the same with the theorem 2.2, i.e. $O\left(\alpha_{2} \log _{2} n\right)$, in which $n$ is the number of nodes in the network. Compared with the centered routing algorithm, such as Dijkstra's shortest path algorithm whose time complexity is $O\left(n^{2}\right), \mathrm{A}^{+}$is efficient shown in fig. 2 .

\section{Conclusions}

The Internet model is built up and distributed routing algorithm A is designed in this paper, whose time complexity is analyzed. The static network model (SNM) and dynamic network model (DNM) are established and path $\mathrm{DB}$ and its replace rules are put forward. The improved distributed routing algorithm $\mathrm{A}^{+}$basded on those models and rules is designed and its time complexity is also analyzed.

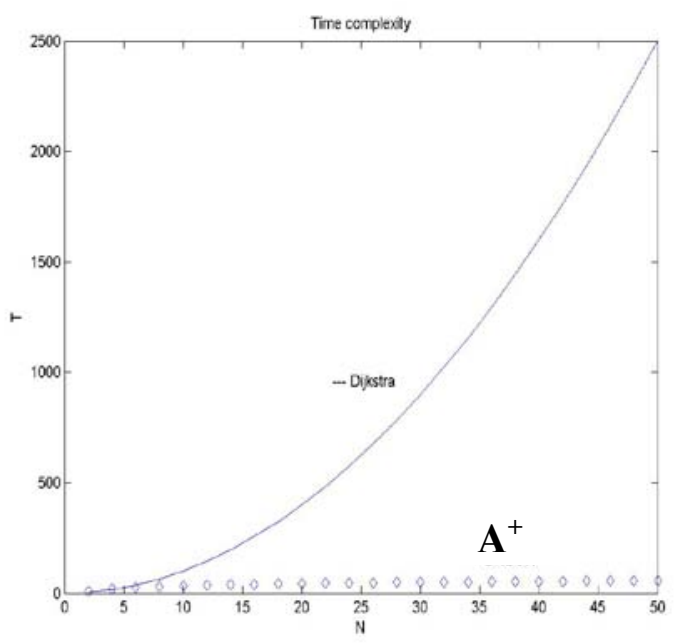

Fig.2. Comparision $\mathrm{A}^{+}$with Dijkstra

\section{References}

[1] Ming Yinghua. Review on computer network routing research [J] , Computer journal. 6(1): 650-662. 2003.

[2] Ming Yinghua. Backward and forward of Computer network [J]. Nature science advances. 10(10): 877-883. 2000.

[3] Feng Jie, Jiang Ning. Distributed QoS routing algorithm based on partheno-GA with binary coding [J], Journal of central south university (science and technology). 38: 159-164. 2007.

[4] Feng Jie, Jiang Ning, Yang Jichun. Routing algorithm with multiple QoS constrains based on multiobjective programming theory [J], Journal of central south university (science and technology). 38: 589-593. 2007.

[5] Feng Jie, Xia Zunqan. Multiple QoS-bounded multicast routing algorithm based on fuzzy set theory, The Proceeding of IFSA2005 World Congress, Springer. Volume III: 1586-1591. 2005.

[6] Feng Jie, study on routing algoritms with QoS constrains based on small \& random graph theory, doctoral dissertation, Dalian university of technology, 2007,10. 